\title{
Alertness and cognitive control: Is there a spatial attention constraint?
}

\author{
Darryl W. Schneider ${ }^{1}$ \\ Published online: 23 October 2018 \\ (C) The Psychonomic Society, Inc. 2018
}

\begin{abstract}
Congruency effects in arrow flanker tasks are often larger when subjects are more alert, suggesting an unusual connection between alertness and cognitive control. Theoretical accounts of the alerting-congruency interaction differ with respect to whether and how spatial attention is involved. In the present study, the author conducted eight experiments to determine whether there is a spatial attention constraint linking alertness to cognitive control. Alertness was manipulated in color-word Stroop tasks involving stimuli that were spatially integrated (Experiments 1-3) or separated (Experiments 4 and 5), as well as in Stroop-like tasks involving spatially separated stimuli for which the irrelevant stimulus features were spatial words (Experiments 6 and 7) or arrows (Experiment 8). All experiments yielded effects of alerting and congruency, but none produced the alerting-congruency interaction typically found with arrow flanker tasks. The results suggest that there is a spatial attention constraint on the relationship between alertness and cognitive control, part of which might involve having a task goal associated with spatial information processing.
\end{abstract}

Keywords Alertness $\cdot$ Cognitive control $\cdot$ Selective attention $\cdot$ Spatial attention $\cdot$ Stroop task

Decades of psychological research have revealed a complex picture of how attention works (for reviews, see Johnson \& Proctor, 2004; Pashler, 1998). Some of the complexity arises from the division of attention into distinct components, two of which are alertness and cognitive control (Fan, McCandliss, Sommer, Raz, \& Posner, 2002; Petersen \& Posner, 2012; Posner \& Boies, 1971). Alertness refers to a fluctuating state of arousal that influences stimulus detection and response readiness. Cognitive control involves the dynamic adjustment of attentional selectivity to modulate information processing in pursuit of a task goal. In the present study, I investigate the generality of a puzzling interaction between alertness and cognitive control.

The starting point for the investigation is the Attention Network Test (ANT) introduced by Fan et al. (2002) for simultaneously studying multiple components of attention. In simplified form, the ANT involves a flanker task (Eriksen \& Eriksen, 1974) in which subjects make a left/right key-press response to classify the left/right direction of a central target arrow flanked by distractor arrows. Response time (RT) is the

Darryl W. Schneider

dws@purdue.edu

1 Department of Psychological Sciences, Purdue University, 703 Third Street, West Lafayette, IN 47907, USA primary dependent measure because error rates are usually low. Alertness is studied by manipulating the presence or absence of an alerting cue (a visual warning signal) prior to the arrow stimuli. RTs are shorter when alerting cues are present (alert trials) than when they are absent (no-alert trials), and this alerting effect is attributed to increased alertness (Posner, 2008). Cognitive control is studied by manipulating the match or mismatch (congruency) between the responses associated with the central target arrow and the flanking distractor arrows. RTs are longer when there is a mismatch (incongruent stimuli; e.g., a left-pointing target arrow flanked by rightpointing distractor arrows) than when there is a match (congruent stimuli; e.g., a left-pointing target arrow flanked by left-pointing distractor arrows). This congruency effect is commonly attributed to cognitive control that reflects a failure of selective attention (i.e., not focusing attention exclusively on the target).

Interestingly, the alerting and congruency manipulations have interactive effects. In several studies involving variants of the ANT, researchers have observed larger congruency effects on alert trials than on no-alert trials (e.g., Callejas, Lupiáñez, Funes, \& Tudela, 2005; Fan et al., 2002; MacLeod et al., 2010; McConnell \& Shore, 2011; Redick \& Engle, 2006; Schneider, 2018a, 2018b; Weinbach \& Henik, 2012). Nieuwenhuis and de Kleijn (2013) noted that this alerting-congruency interaction is counterintuitive because 
its form is opposite that of a normal scaling effect, which would be characterized by smaller-not larger-effect sizes at shorter RTs. Scaling effects would be expected because of the positive linear relationship between the mean and standard deviation $(S D)$ of an RT distribution (Wagenmakers \& Brown, 2007), as well as the observation that standard models of decision-making naturally produce larger effect sizes at longer RTs based on the geometry of the decision process (Smith \& Ratcliff, 2015). Thus, the finding of a larger congruency effect in the context of shorter overall RTs on alert trials than on noalert trials suggests an unusual connection between alertness and cognitive control, such that being more alert impairs selective attention and allows for more influence of distractors.

Most of the theoretical accounts of the alerting-congruency interaction can be divided into two groups. The first group consists of explanations that link alertness to cognitive control via mechanisms related to spatial attention. An idea shared by some of these accounts is that spatial attention is more diffuse on alert trials than on no-alert trials (McConnell \& Shore, 2011; Nieuwenhuis \& de Kleijn, 2013; Weinbach \& Henik, 2012), which might improve stimulus detection or encoding (resulting in short overall RTs) while giving more attention to distractors in the decision process (resulting in large congruency effects).

Weinbach and Henik (2012) suggested that alerting cues directly trigger diffuse attention in order to increase the accessibility of spatial information in the visual field. This idea is consistent with evidence that increased alertness induces a bias toward global processing of stimuli (Weinbach \& Henik, 2011; but see Weinbach \& Henik, 2014) and that alerting cues improve spatial attention in patients with unilateral neglect (Robertson, Mattingley, Rorden, \& Driver, 1998). In contrast, Nieuwenhuis and de Kleijn (2013) proposed an indirect link between alertness and cognitive control based on diffuse attention. They assumed spatial attention is initially diffuse regardless of trial type, then it is focused on the target as the trial progresses, consistent with conditional accuracy functions in behavioral data (Gratton, Coles, Sirevaag, Eriksen, \& Donchin, 1988; Heitz \& Engle, 2007) and with how selective attention works in some models of the flanker task (Spencer \& Coles, 1999; White, Ratcliff, \& Starns, 2011). Increased alertness is assumed to shorten stimulus encoding time (see Rolke \& Hofmann, 2007; Seifried, Ulrich, Bausenhart, Rolke, \& Osman, 2010), allowing the decision process to start earlier on alert trials - when attention is less focused on the target - than on no-alert trials. ${ }^{1}$ Schneider (2018b) proposed an alternative to diffuse attention, suggesting that alertness affects how stimuli are spatially grouped into

\footnotetext{
$\overline{1}$ The relative timing of cognitive processes on alert versus no-alert trials might also allow mechanisms such as arousal-biased competition (Mather \& Sutherland, 2011) to accommodate the alerting-congruency interaction (Warren, Murphy, \& Nieuwenhuis, 2016).
}

object representations within the focus of attention. Greater alertness is assumed to lower the spatial grouping threshold (see Logan, 1996) and make it more likely that distractors are grouped with the target in the object representations selected for analysis, resulting in more distractor processing on alert trials than on no-alert trials. In all of the accounts in this group, spatial attention plays an important role in explaining how alertness is related to cognitive control.

The second group of explanations for the alerting-congruency interaction does not involve mechanisms that are closely related to spatial attention. Instead, these accounts draw on ideas about inhibition or activation to link alerting and cognitive control processes (Callejas et al., 2005; Callejas, Lupiáñez, \& Tudela, 2004; Fischer, Plessow, \& Kiesel, 2010, 2012), such that increased alertness helps stimulus encoding (resulting in short overall RTs) in ways that attenuate control of the decision process (resulting in large congruency effects).

Callejas and colleagues suggested that increased alertness leads to inhibition of cognitive control, enabling faster stimulus detection at the expense of reduced discrimination between the target and distractors (Callejas et al., 2005; Callejas et al., 2004). As an alternative to inhibition, Fischer et al. (2010, 2012; see also Böckler, Alpay, \& Stürmer, 2011) proposed that increased alertness facilitates stimulus encoding and boosts activation of an uncontrolled, direct route for response selection, whereby stimuli activate responses based on established stimulus-response associations. Strong activation from distractors would exacerbate their influence on response selection, resulting in a larger congruency effect on alert trials than on no-alert trials. For the accounts in this group, none of the mechanisms depend on spatial attention for explaining the alerting-congruency interaction, although they do not rule out a role for it. ${ }^{2}$

The preceding summary of theoretical accounts raises an important question: Is there a spatial attention constraint on the relationship between alertness and cognitive control? It is unclear from previous research whether the alerting-congruency interaction is found only in contexts that involve spatial attention in one form or another, primarily because the interaction is often obtained using close variants of the original

\footnotetext{
${ }^{2}$ Weinbach and Henik (2014) proposed a saliency account of the alertingcongruency interaction that does not fit neatly into either of the two groups I discussed. Using a global/local task paradigm (Navon, 1977) involving hierarchically organized arrow stimuli, they found typical alerting-congruency interactions only when the irrelevant stimulus dimension was perceptually salient. This finding led them to suggest that increased alertness enhances sensitivity to salient information. On the one hand, the saliency account does not require a role for spatial attention (e.g., the local stimulus dimension was made more salient in their experiment by presenting arrows in different colors, which is a nonspatial manipulation). On the other hand, the evidence supporting the saliency account comes from a paradigm in which spatial attention can vary between the global and local dimensions of stimuli that convey spatial information. Thus, the importance of spatial attention for the saliency account is unclear.
} 
ANT (Fan et al., 2002). The arrow flanker task at the heart of the ANT includes spatial separation of the target and distractors, stimuli (arrows) that have well-established spatial connotations, and spatially defined responses (left and right key presses). Considering the role of spatial attention in some of the aforementioned theoretical accounts, it is potentially informative to know whether the alerting-congruency interaction generalizes beyond the arrow flanker task to other tasks that putatively involve cognitive control and yield congruency effects, but do not involve spatial attention to the same extent.

One of the alternative tasks in which alertness and cognitive control have been examined is the Simon task (Simon \& Small, 1969). In a typical version of the Simon task (for a review, see Lu \& Proctor, 1995), subjects make a left/right key-press response to classify a nonspatial feature (e.g., color) of a stimulus that is presented at a varying spatial location (left or right of fixation) across trials. Cognitive control is studied by manipulating the match or mismatch between the spatial location of the stimulus and response. RTs are longer when there is a mismatch (incongruent stimuli; e.g., a green stimulus - associated with a left response - presented on the right) than when there is a match (congruent stimuli; e.g., a red stimulus - associated with a right response - presented on the right). This congruency effect (also known as the Simon effect) is commonly attributed to cognitive control that reflects a failure of selective attention - in this case, not ignoring an irrelevant stimulus feature (spatial location) when selecting a spatial response to the relevant stimulus feature (color).

Alerting effects on Simon task performance have been examined in a few studies (Böckler et al., 2011; Fischer et al., 2010; Klein \& Ivanoff, 2011; Soutschek, Müller, \& Schubert, 2013), all of which revealed alerting-congruency interactions analogous to those typically obtained with the arrow flanker task. These results indicate that the interaction is not unique to the arrow flanker task and can be found when the relevant and irrelevant stimulus features are not spatially separated. However, they do not answer the question of whether there is a spatial attention constraint because the mere occurrence of congruency effects in the Simon task indicates that subjects shift attention to and process the spatial location of the stimulus.

Another alternative task for studying alertness and cognitive control is the Stroop task (Stroop, 1935). In a typical color-word version of the Stroop task (for a review, see MacLeod, 1991), subjects make a manual or vocal response to classify the physical color of a color word. Cognitive control is studied by manipulating the match or mismatch between the physical color and word meaning. RTs are longer when there is a mismatch (incongruent stimuli; e.g., the word $R E D$ presented in green) than when there is a match (congruent stimuli; e.g., the word RED presented in red). This congruency effect (also known as the Stroop effect) is commonly attributed to cognitive control that reflects a failure of selective attention-in this case, not ignoring an irrelevant stimulus feature (word meaning) when selecting a response to the relevant stimulus feature (physical color).

The color-word Stroop task seems to be a good candidate for assessing whether there is a spatial attention constraint linking alertness to cognitive control because there is no spatial separation of relevant and irrelevant stimulus features (unlike in the flanker task) and the stimuli do not have wellestablished spatial connotations (unlike arrow stimuli in the flanker task or stimulus locations in the Simon task). However, to the best of my knowledge, alerting effects on color-word Stroop task performance have been examined in only two experiments (Soutschek et al., 2013, Experiment 2, $N=30$; Weinbach \& Henik, 2012, Experiment 2, $N=14$ ), neither of which revealed a significant alerting-congruency interaction. These results hint at a spatial attention constraint, but I do not think a firm conclusion can be drawn from null interactions in two experiments with modest sample sizes. Considering concerns about replicability in psychology (e.g., Open Science Collaboration, 2015), it seemed worthwhile to determine whether these previous findings of null alertingcongruency interactions in the Stroop task can be replicated in higher-powered experiments. If the findings are replicable, then the stage would be set for exploring the nature of the spatial attention constraint.

I started the present study with a conceptual replication of Experiment 2 from Weinbach and Henik (2012). Their subjects performed a color-word Stroop task that involved making a key-press response to classify the physical color (green or red) of a letter string (the Hebrew words for GREEN or $R E D$, or $X X X X$ as a neutral stimulus). On no-alert trials, the task stimulus was presented after a constant fixation interval. On alert trials, an auditory alerting cue sounded briefly during the fixation interval, shortly before the onset of the task stimulus. There were significant effects of alerting and congruency, but nearly identical congruency effects on alert and noalert trials, resulting in a nonsignificant alerting-congruency interaction. Weinbach and Henik favored an interpretation of their results that implied a spatial attention constraint, arguing that the interaction depends on the extent to which a task involves spatial attention.

In Experiment 1 of the present study, subjects performed a color-word Stroop task that involved making a left/right keypress response to classify the physical color (green or red) of a color word (the English words GREEN or RED). An example stimulus is shown in Fig. 1. I modified the experiment design of Weinbach and Henik (2012) in three main respects (besides changing the language of the stimulus words). First, I did not include a neutral stimulus because Weinbach and Henik observed similar performance on congruent and neutral trials, consistent with the finding that congruency effects in the Stroop task mostly reflect interference on incongruent trials (e.g., Dunbar \& MacLeod, 1984). Second, I used a variable 


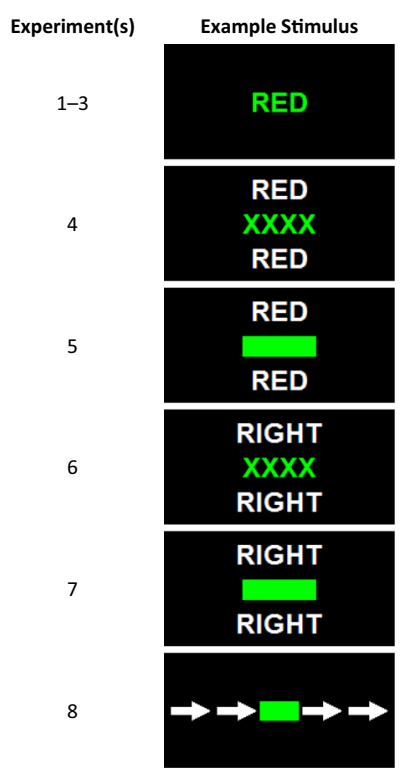

Fig. 1 Example stimulus for each experiment. For each example, the target color is green and the stimulus is incongruent. It is assumed that green and red colors are mapped to left-key and right-key responses, respectively

fixation interval prior to the color-word stimulus, which is a common aspect of the ANT (Fan et al., 2002). Third, my alerting cue was visual (a square presented briefly at fixation) instead of auditory, which more closely matches how alerting is often implemented in the ANT and precludes modality switch effects that might complicate the interpretation of any alerting-congruency interaction. ${ }^{3}$ In published (Schneider, 2018a) and unpublished (Schneider, 2018c) experiments from my laboratory, this kind of alerting cue has consistently elicited typical alerting-congruency interactions in arrow flanker tasks.

Considering the design modifications, I regard my Experiment 1 as a conceptual replication rather than a direct replication of Experiment 2 in Weinbach and Henik (2012). ${ }^{4}$ For ease of exposition, I report Experiment 1 in conjunction with my Experiments 2 and 3, all of which were identical except for

\footnotetext{
${ }^{3}$ Reliable alerting-congruency interactions have been obtained in previous studies involving auditory alerting cues and visual stimuli (e.g., Callejas et al., 2004; Fischer et al., 2012; Weinbach \& Henik, 2012). However, the use of auditory alerting cues creates a confound: attention switches between modalities on alert trials but not on no-alert trials. There is evidence that modality switching generates performance costs (e.g., Lukas, Philipp, \& Koch, 2010; Quinlan \& Hill, 1999), although it is unknown what effect (if any) it has on the alerting-congruency interaction. I avoided the confound by using visual alerting cues in my experiments.

${ }^{4}$ Experiments 1 and 8 were preregistered conceptual replications of Experiments 2 and 4, respectively, from Weinbach and Henik (2012). The protocols are publicly available at https://aspredicted.org/9jq4y.pdf (Experiment 1) and https://aspredicted.org/jy9bn.pdf (Experiment 8). Experiments 2-7 were not preregistered, but they adhered closely to key aspects of the protocols for Experiments 1 and 8 (e.g., sample size, experiment design, trial procedure, data exclusion criteria, and statistical analyses). All experiments that were conducted for the present study are reported in this article.
}

the external reminders to subjects of how colors were mapped to responses. In Experiment 1, lights above the response keys were illuminated in the colors mapped to the responses, similar to the reminders used by Weinbach and Henik (color patches on the keys). However, there is evidence that congruency effects in the Stroop task are smaller when the response keys are labeled with physical colors than with color words (McClain, 1983). Consequently, I changed the external reminders for Experiments 2 and 3 in an effort to amplify the overall congruency effects. In Experiment 2, there were no external reminders. In Experiment 3, there were word-based external reminders (e.g., "left key: green" and "right key: red") in the bottom corners of the display monitors. The results were similar across experiments, suggesting that the type of external reminder was relatively inconsequential. More importantly, the three experiments provided replicated data patterns concerning whether the alertingcongruency interaction typically found in the arrow flanker task occurs in the color-word Stroop task.

\section{Experiments 1-3}

\section{Method}

Subjects Each experiment involved 86 students from Purdue University who participated for course credit (Experiment 1: 51 female, 80 right-handed, mean age $=18.7$ years; Experiment 2: 40 female, 78 right-handed, mean age $=19.3$ years; Experiment 3: 40 female, 81 right-handed, mean age $=18.9$ years). All subjects in the present study came from the same university population, and none participated in more than one experiment. All subjects whose data were analyzed reported having normal or corrected-to-normal vision and no color blindness. ${ }^{5}$ The preregistered sample size was based on power analyses involving mean RTs from Experiment 2 of Weinbach and Henik (2012) and estimates of standard deviations and correlations between conditions derived from Experiment 1 of Schneider (2018b). Simulations indicated that 85 subjects would provide $80 \%$ power to detect an alerting-congruency interaction reflecting a $12-\mathrm{ms}$ difference in congruency effects on RTs between alert and noalert trials $(d=0.3)$, which is approximately $50 \%$ of the difference obtained for the significant alerting-congruency interaction found with an arrow flanker task in Experiment 1 of Weinbach and Henik (2012). The sample size was set to 86 to allow for equal counterbalancing of color-response mappings across subjects.

\footnotetext{
${ }^{5}$ Across the eight experiments in the present study, data were excluded from seven additional subjects (one in Experiment 2, three in Experiment 4, and three in Experiment 6) who met preregistered exclusion criteria: Four of the subjects had mean error rates exceeding 20\% and three of the subjects had mean RTs exceeding four standard deviations of their respective group means. Six additional subjects were replaced (their data were not analyzed) because they reported some form of colorblindness or uncorrected vision impairment.
} 
Apparatus All experiments in the present study were conducted on computers that displayed stimuli on monitors with a black background $\left(0.19 \mathrm{~cd} / \mathrm{m}^{2}\right.$; RGB color code: $\left.0,0,0\right)$ at an uncontrolled viewing distance of approximately $50 \mathrm{~cm}$. Responses were registered from the left-most and right-most keys (pressed with the left and right index fingers, respectively) of Chronos devices (Psychology Software Tools, Inc.). Light-emitting diodes (LEDs) located above the response keys were illuminated continuously throughout each experiment.

Task and stimuli Subjects performed a color-word Stroop task that involved classifying the physical color of a color word by pressing a designated response key. The physical colors were green $\left(101.1 \mathrm{~cd} / \mathrm{m}^{2}\right.$; RGB color code: $\left.0,255,0\right)$ and red $(24.0$ $\mathrm{cd} / \mathrm{m}^{2}$; RGB color code: $255,0,0$ ), and the color words were GREEN and RED. Each color-word stimulus appeared in bold 18-point Arial font, measuring $0.4 \mathrm{~cm}$ high and either $1.2 \mathrm{~cm}$ $(R E D)$ or $2.0 \mathrm{~cm}(G R E E N)$ wide. All four possible combinations of the physical color and meaning of the word constituting each color-word stimulus were used, resulting in stimuli that were either congruent (physical color and word meaning matched; e.g., red-colored RED) or incongruent (physical color and word meaning mismatched; e.g., green-colored $R E D$; see Fig. 1). In addition to the color-word stimuli, all trials included a white fixation cross $(0.35 \mathrm{~cm} \times 0.35 \mathrm{~cm} ; 135.8$ $\mathrm{cd} / \mathrm{m}^{2}$; RGB color code: $\left.255,255,255\right)$, and alert trials included an alerting cue (a white outlined square measuring $0.7 \mathrm{~cm} \times$ $0.7 \mathrm{~cm} ; 135.8 \mathrm{~cd} / \mathrm{m}^{2}$; RGB color code: $\left.255,255,255\right)$.

Procedure All subjects in the present study were tested individually after providing informed consent for a study protocol approved by the Purdue University Institutional Review Board. Instructions were presented onscreen and read aloud by the experimenter. Subjects were instructed to identify the color of each color-word stimulus while ignoring the meaning of the word, trying their best to respond quickly and accurately. Depending on the experiment (as described later), they were provided with external reminders of the color-response mappings. Subjects performed eight example no-alert trials (two trials for each color-word stimulus) with accuracy feedback during the instructions. Afterward, they completed 10 blocks of 32 trials per block without accuracy feedback.

Each trial started with a fixation cross presented centrally for a randomly selected interval of $1,000 \mathrm{~ms}, 1,500 \mathrm{~ms}, 2,000 \mathrm{~ms}$, or $2,500 \mathrm{~ms}$, after which time the fixation cross was replaced by a color-word stimulus that remained visible until a response key was pressed. After the response was registered, the stimulus disappeared, and the variable fixation interval for the next trial began immediately. No-alert and alert trials were identical, except that on the latter an alerting cue was presented $500 \mathrm{~ms}$ before the color-word stimulus, surrounding the fixation cross for $100 \mathrm{~ms}$ before disappearing. All possible combinations of fixation interval, alerting, and color-word stimulus occurred equally often in random order in every block of trials. The mappings of colors (green and red) to responses (left and right keys) were counterbalanced across subjects in each experiment.

The only differences between Experiments 1-3 concerned the external reminders of the color-response mappings. In Experiment 1, the LEDs located above the response keys were illuminated in the colors mapped to the responses, providing external reminders of the mappings (e.g., if green and red were mapped to left and right keys, respectively, then the LEDs above those keys were colored green and red). In Experiment 2, the LEDs above both response keys were white, providing no external reminders of the color-response mappings. In Experiment 3 (and in all subsequent experiments in the present study), the LEDs above both response keys were white, but word-based external reminders (e.g., "left key: green" and "right key: red") appeared in the bottom corners of the computer screen for the duration of the experiment.

\section{Results}

Data trimming and statistical analyses followed the preregistered protocol. The first block of trials was treated as practice and excluded. Trials with RTs more than three standard deviations above the mean in each condition for a given subject were excluded ( $\leq 2.0 \%$ of trials for every experiment in the present study). Error trials were excluded from the RT analyses. Mean RTs and error rates as a function of alerting (no alert or alert) and congruency (incongruent or congruent) appear in Table 1. Those variables were factors in repeatedmeasures analyses of variance (ANOVAs) with $\alpha=.05$. The ANOVA results are summarized in Table 2. Regardless of statistical significance, alerting-congruency interactions were evaluated further with Bayes-factor analyses involving scaled JZS Bayes factors with $r=1$ (Rouder, Speckman, Sun, Morey, \& Iverson, 2009). ${ }^{6}$ For all experiments in the present study, the error data patterns were consistent with the RT data patterns and there were no indications of speed-accuracy trade-

\footnotetext{
${ }^{6}$ Every reported Bayes factor $(B F)$ has a subscript (null or alt) indicating whether the null or nondirectional alternative hypothesis is favored by the data for the alerting-congruency interaction effect. The favored hypothesis corresponds to the hypothesis under which the data are more likely. For example, $B F_{\text {null }}=12$ indicates that the data are 12 times more likely under the null hypothesis of no difference in congruency effects between alert and no-alert trials (i.e., no interaction) than the alternative hypothesis of a difference (i.e., an interaction). As another example, $B F_{\text {alt }}=4$ indicates that the data are 4 times more likely under the alternative hypothesis than the null hypothesis. Researchers have proposed various schemes for categorizing Bayes factors into levels of evidence (e.g., Held \& Ott, 2018; Schönbrodt \& Wagenmakers, 2018). Consistent with these schemes, I interpret Bayes factors in relation to the strength of evidence for the favored hypothesis as follows: 1 (no evidence); 1-3 (weak evidence); 3-10 (moderate evidence); 10-30 (strong evidence); $30-100$ (very strong evidence); and $>100$ (decisive evidence). Returning to the examples, $B F_{\text {null }}=12$ indicates strong evidence for the null hypothesis (i.e., the absence of an alerting-congruency interaction), and $B F_{\text {alt }}$ $=4$ indicates moderate evidence for the alternative hypothesis (i.e., the presence of an alerting-congruency interaction).
} 
Table 1 Mean response times and error rates

\begin{tabular}{|c|c|c|c|c|}
\hline Experiment & Alerting & Congruency & $\begin{array}{l}\text { Response time } \\
(\mathrm{ms})\end{array}$ & Error rate $(\%)$ \\
\hline \multirow[t]{4}{*}{1} & \multirow[t]{2}{*}{ No alert } & Incongruent & $529(11)$ & $2.4(.2)$ \\
\hline & & Congruent & $503(9)$ & $2.0(.3)$ \\
\hline & \multirow[t]{2}{*}{ Alert } & Incongruent & $492(11)$ & $2.2(.3)$ \\
\hline & & Congruent & $466(8)$ & $1.4(.2)$ \\
\hline \multirow[t]{4}{*}{2} & \multirow[t]{2}{*}{ No alert } & Incongruent & $521(10)$ & $3.7(.4)$ \\
\hline & & Congruent & $498(8)$ & $2.7(.3)$ \\
\hline & \multirow[t]{2}{*}{ Alert } & Incongruent & $479(10)$ & $3.0(.4)$ \\
\hline & & Congruent & $459(9)$ & $2.1(.3)$ \\
\hline \multirow[t]{4}{*}{3} & \multirow[t]{2}{*}{ No alert } & Incongruent & $552(15)$ & $4.0(.4)$ \\
\hline & & Congruent & $509(11)$ & $2.1(.2)$ \\
\hline & \multirow[t]{2}{*}{ Alert } & Incongruent & $516(14)$ & $3.4(.3)$ \\
\hline & & Congruent & $474(10)$ & $1.6(.2)$ \\
\hline \multirow[t]{4}{*}{4} & \multirow[t]{2}{*}{ No alert } & Incongruent & $504(10)$ & $3.5(.4)$ \\
\hline & & Congruent & $488(8)$ & $3.0(.3)$ \\
\hline & \multirow[t]{2}{*}{ Alert } & Incongruent & $469(10)$ & $2.5(.3)$ \\
\hline & & Congruent & $456(9)$ & $1.9(.2)$ \\
\hline \multirow[t]{4}{*}{5} & \multirow[t]{2}{*}{ No alert } & Incongruent & $516(11)$ & $3.6(.4)$ \\
\hline & & Congruent & $494(9)$ & $2.3(.3)$ \\
\hline & \multirow[t]{2}{*}{ Alert } & Incongruent & $462(9)$ & $2.4(.3)$ \\
\hline & & Congruent & $449(8)$ & $1.7(.2)$ \\
\hline \multirow[t]{4}{*}{6} & \multirow[t]{2}{*}{ No alert } & Incongruent & $518(10)$ & $3.9(.4)$ \\
\hline & & Congruent & $495(9)$ & $2.0(.3)$ \\
\hline & \multirow[t]{2}{*}{ Alert } & Incongruent & $473(10)$ & $3.2(.3)$ \\
\hline & & Congruent & $461(8)$ & $1.6(.2)$ \\
\hline \multirow[t]{4}{*}{7} & \multirow[t]{2}{*}{ No alert } & Incongruent & $520(10)$ & $4.7(.6)$ \\
\hline & & Congruent & $495(9)$ & $2.3(.4)$ \\
\hline & \multirow[t]{2}{*}{ Alert } & Incongruent & $472(10)$ & $3.5(.4)$ \\
\hline & & Congruent & $451(9)$ & $1.7(.3)$ \\
\hline \multirow[t]{4}{*}{8} & \multirow[t]{2}{*}{ No alert } & Incongruent & $495(9)$ & $3.7(.4)$ \\
\hline & & Congruent & $462(8)$ & $1.5(.3)$ \\
\hline & \multirow[t]{2}{*}{ Alert } & Incongruent & $464(10)$ & $3.3(.4)$ \\
\hline & & Congruent & $428(9)$ & $.9(.2)$ \\
\hline
\end{tabular}

Note. Standard errors of the means appear in parentheses

offs (see Tables 1 and 2); therefore, I focused on the RT data, which are presented visually in Fig. 2.

Experiment 1 Subjects were faster on alert trials (mean RT of $479 \mathrm{~ms}$ ) than on no-alert trials $(516 \mathrm{~ms})$, resulting in a significant main effect of alerting. They were slower on incongruent trials $(510 \mathrm{~ms})$ than on congruent trials $(485 \mathrm{~ms})$, resulting in a significant main effect of congruency. Congruency effects were $25 \mathrm{~ms}$ and $26 \mathrm{~ms}$ for alert and no-alert trials, respectively, resulting in a nonsignificant interaction, $B F_{\text {null }}=11.7$.

Experiment 2 Subjects were faster on alert trials ( $469 \mathrm{~ms}$ ) than on no-alert trials $(510 \mathrm{~ms})$, resulting in a significant main effect of alerting. They were slower on incongruent trials $(500 \mathrm{~ms})$ than on congruent trials $(479 \mathrm{~ms})$, resulting in a significant main effect of congruency. Congruency effects were $20 \mathrm{~ms}$ and $23 \mathrm{~ms}$ for alert and no-alert trials, respectively, resulting in a nonsignificant interaction, $B F_{\text {null }}=9.2$.

Experiment 3 Subjects were faster on alert trials ( $495 \mathrm{~ms}$ ) than on no-alert trials $(530 \mathrm{~ms})$, resulting in a significant main effect of alerting. They were slower on incongruent trials (534 ms) than on congruent trials $(491 \mathrm{~ms})$, resulting in a significant main effect of congruency. Congruency effects were $42 \mathrm{~ms}$ and $43 \mathrm{~ms}$ for alert and no-alert trials, respectively, resulting in a nonsignificant interaction, $B F_{\text {null }}=11.7$.

\section{Discussion}

Experiments 1-3 produced similar patterns of results (see Fig. 2). Subjects were faster on alert trials than on no-alert trials, indicating that the visual alerting cues were effective for increasing alertness. This is consistent with previous experiments in which alerting effects were induced by visual cues (e.g., Fan et al., 2002; McConnell \& Shore, 2011; Redick \& Engle, 2006; Schneider, 2018a, 2018b). Subjects were slower on incongruent trials than on congruent trials, indicating that the meaning of the word - which was irrelevant and could be ignored - was processed nevertheless and influenced task performance. This is consistent with previous experiments demonstrating congruency effects in the color-word Stroop task (for a review, see MacLeod, 1991). Critically, congruency effects were similar on alert and no-alert trials in all three experiments, resulting in nonsignificant alerting-congruency interactions and Bayes factors that generally indicated strong evidence of no differences in congruency effects. These findings replicate the null interactions obtained in two previous experiments (Soutschek et al., 2013; Weinbach \& Henik, 2012), but with larger sample sizes that provided higher statistical power for detecting interactions.

These results establish that the alerting-congruency interaction typically found in the arrow flanker task does not occur in the color-word Stroop task. This is important at a theoretical level because it indicates that the underlying mechanism by which alertness influences cognitive control does not generalize across all tasks that putatively involve cognitive control. This lack of generality is problematic for theoretical accounts that link alertness to cognitive control by inhibition or activation mechanisms (e.g., Callejas et al., 2005; Fischer et al., 2010, 2012). If increased alertness leads to inhibition of cognitive control, then alerting-congruency interactions should have been obtained for the Stroop task in Experiments 1-3 because it is widely considered to involve cognitive control. This expectation also holds for the idea that increased alertness boosts activation of a direct route for response selection involving established stimulus-response associations. Word 
Table 2 Summary of analyses of variance

\begin{tabular}{|c|c|c|c|c|c|c|c|c|c|}
\hline \multirow[t]{2}{*}{ Exp. } & \multirow[t]{2}{*}{ Effect } & \multicolumn{4}{|c|}{ Response time } & \multicolumn{4}{|l|}{ Error rate } \\
\hline & & $F(1,85)$ & $M S E$ & $p$ & $\eta_{\mathrm{p}}^{2}$ & $F(1,85)$ & $M S E$ & $p$ & $\eta_{\mathrm{p}}^{2}$ \\
\hline \multirow[t]{3}{*}{1} & A & 140.22 & 833 & $<.001$ & .623 & 6.29 & 2 & .014 & .069 \\
\hline & $\mathrm{C}$ & 49.77 & 1,128 & $<.001$ & .369 & 6.46 & 5 & .013 & .071 \\
\hline & $\mathrm{A} \times \mathrm{C}$ & $<.01$ & 471 & .960 & $<.001$ & 1.43 & 4 & .235 & .017 \\
\hline \multirow[t]{3}{*}{2} & A & 256.73 & 546 & $<.001$ & .751 & 9.54 & 4 & .003 & .101 \\
\hline & $\mathrm{C}$ & 40.66 & 951 & $<.001$ & .324 & 10.12 & 7 & .002 & .106 \\
\hline & $\mathrm{A} \times \mathrm{C}$ & .50 & 441 & .482 & .006 & .07 & 4 & .795 & .001 \\
\hline \multirow[t]{3}{*}{3} & A & 74.44 & 1,432 & $<.001$ & .467 & 7.82 & 3 & .006 & .084 \\
\hline & $\mathrm{C}$ & 60.40 & 2,588 & $<.001$ & .415 & 39.76 & 7 & $<.001$ & .319 \\
\hline & $\mathrm{A} \times \mathrm{C}$ & .01 & 650 & .905 & $<.001$ & .07 & 4 & .787 & .001 \\
\hline \multirow[t]{3}{*}{4} & A & 128.78 & 749 & $<.001$ & .602 & 23.29 & 4 & $<.001$ & .215 \\
\hline & $\mathrm{C}$ & 26.67 & 680 & $<.001$ & .239 & 5.97 & 5 & .017 & .066 \\
\hline & $\mathrm{A} \times \mathrm{C}$ & .28 & 681 & .600 & .003 & .10 & 4 & .758 & .001 \\
\hline \multirow[t]{3}{*}{5} & A & 219.06 & 972 & $<.001$ & .720 & 19.94 & 3 & $<.001$ & .190 \\
\hline & $\mathrm{C}$ & 29.35 & 931 & $<.001$ & .257 & 13.31 & 7 & $<.001$ & .135 \\
\hline & $\mathrm{A} \times \mathrm{C}$ & 4.49 & 427 & .037 & .050 & 3.04 & 3 & .085 & .035 \\
\hline \multirow[t]{3}{*}{6} & A & 214.67 & 638 & $<.001$ & .716 & 6.34 & 4 & .014 & .069 \\
\hline & $\mathrm{C}$ & 28.96 & 891 & $<.001$ & .254 & 22.99 & 11 & $<.001$ & .213 \\
\hline & $\mathrm{A} \times \mathrm{C}$ & 6.91 & 364 & .010 & .075 & .46 & 5 & .499 & .005 \\
\hline \multirow[t]{3}{*}{7} & A & 173.80 & 1,063 & $<.001$ & .672 & 16.60 & 4 & $<.001$ & .163 \\
\hline & $\mathrm{C}$ & 39.96 & 1,106 & $<.001$ & .320 & 37.74 & 10 & $<.001$ & .308 \\
\hline & $\mathrm{A} \times \mathrm{C}$ & .92 & 426 & .342 & .011 & 1.76 & 4 & .188 & .020 \\
\hline \multirow[t]{3}{*}{8} & A & 89.52 & 1,033 & $<.001$ & .513 & 4.88 & 4 & .030 & .054 \\
\hline & $\mathrm{C}$ & 153.37 & 671 & $<.001$ & .643 & 79.78 & 6 & $<.001$ & .484 \\
\hline & $\mathrm{A} \times \mathrm{C}$ & .47 & 335 & .496 & .005 & .28 & 3 & .596 & .003 \\
\hline
\end{tabular}

Note. Exp. = experiment; $\mathrm{A}=$ alerting; $\mathrm{C}=$ congruency

reading is considered to be a fast and automatic process based on well-learned stimulus-response associations, which is why word meaning influences Stroop task performance in the first place (MacLeod, 1991). If increased alertness facilitates this kind of processing, then the irrelevant word part of the colorword stimulus should have been processed to a greater extent-producing a larger congruency effect —on alert trials than on no-alert trials, but that did not happen. The absence of alerting-congruency interactions in Experiments 1-3 supports theoretical accounts that involve some form of spatial attention constraint on the relationship between alertness and cognitive control - a constraint that is present in the arrow flanker task but not in the color-word Stroop task.

What is the nature of the spatial attention constraint? I attempted to answer this question in the remaining experiments by investigating ways in which the color-word Stroop task differs from the arrow flanker task with respect to spatial attention and spatial information processing. A salient difference between the tasks is that the relevant and irrelevant stimulus features are spatially integrated in the Stroop task (the physical color and the word constituting each color-word stimulus occupy the same spatial position), whereas they are spatially separated in the flanker task (the target and distractors occupy different spatial positions). If the alertingcongruency interaction in the flanker task arises from diffuse attention (McConnell \& Shore, 2011; Nieuwenhuis \& de Kleijn, 2013; Weinbach \& Henik, 2012) or spatial grouping (Schneider, 2018b), then one would not necessarily expect the interaction to occur with spatially integrated stimuli, because neither mechanism should modulate selective attention between relevant and irrelevant stimulus features occupying the same space. ${ }^{7}$ This raises the possibility that the alertingcongruency interaction might occur in the Stroop task if the stimulus features were spatially separated.

\footnotetext{
${ }^{7}$ This expectation might seem inconsistent with the aforementioned findings of alerting-congruency interactions in the Simon task (Böckler et al., 2011; Fischer et al., 2010; Klein \& Ivanoff, 2011; Soutschek et al., 2013), in which the relevant and irrelevant stimulus features are spatially integrated. However, given that the irrelevant feature is the spatial location of the stimulus, it is not obvious that findings in the Simon task would generalize to the Stroop task. A precedent for this point is the finding of different time courses for the congruency effects in the Simon and Stroop tasks (e.g., Pratte, Rouder, Morey, \& Feng, 2010).
} 

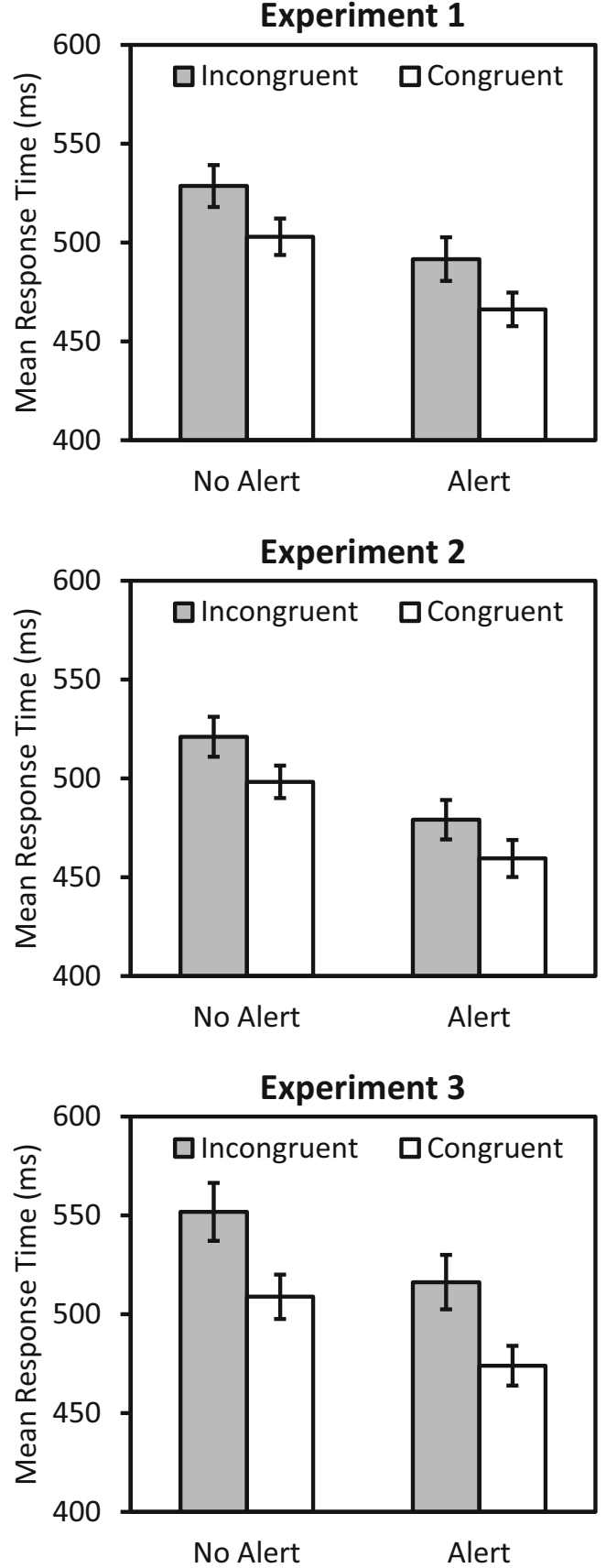

Fig. 2 Mean response times as a function of alerting and congruency in Experiments 1-3. Error bars represent standard errors of the means

In Experiments 4 and 5, subjects performed a color-word Stroop task that involved making a left/right key-press response to classify the physical color (green or red) of a target stimulus that was flanked above and below by distractors that were color words (GREEN or RED). The target stimulus was a colored letter string $(X X X X)$ in Experiment 4 and a colored rectangle in Experiment 5. An example stimulus for each experiment is shown in Fig. 1. The distractor words were always displayed in a neutral color (white). Thus, the relevant and irrelevant stimulus features were still physical color and word meaning, respectively, but they were spatially separated. Experiments 4 and 5 were otherwise identical to Experiment 3 (including the word-based external reminders of the colorresponse mappings). If increased alertness is associated with more diffuse attention or more spatial grouping of targets and distractors, then there is an opportunity for those spatial attention mechanisms to modulate Stroop task performance involving spatially separated stimuli, possibly resulting in alertingcongruency interactions.

\section{Experiments 4 and 5}

\section{Method}

Subjects Each experiment involved 86 students (Experiment 4: 41 female, 74 right-handed, mean age $=19.0$ years; Experiment 5: 63 female, 76 right-handed, mean age $=19.1$ years).

Task and stimuli Subjects performed a color-word Stroop task that involved classifying the physical color (green or red) of a centrally presented target stimulus that was flanked by identical distractor color words (GREEN or RED) above and below it. The physical color of the distractor words was always white. In Experiment 4, the target stimulus was a colored letter string $(X X X X)$, whereas in Experiment 5, it was a colored rectangle. Each target stimulus was $0.4 \mathrm{~cm}$ high and $1.5 \mathrm{~cm}$ wide. The center-to-center distance between the target stimulus and each distractor word was $0.7 \mathrm{~cm}$. All four possible combinations of target color and distractor word were used, resulting in stimulus displays that were either congruent (target color and distractor word meaning matched; e.g., red $X X X X$ or red rectangle flanked by $R E D$ ) or incongruent (target color and distractor word meaning mismatched; e.g., green $X X X X$ or green rectangle flanked by $R E D$; see Fig. 1).

Procedure The procedure was identical to that of Experiment 3.

\section{Results}

Data trimming and statistical analyses followed those of Experiments 1-3. Mean RTs and error rates as a function of alerting and congruency appear in Table 1 . The ANOVA results are summarized in Table 2. The RT data are presented visually in Fig. 3.

Experiment 4 Subjects were faster on alert trials $(463 \mathrm{~ms})$ than on no-alert trials $(496 \mathrm{~ms})$, resulting in a significant main effect of alerting. They were slower on incongruent trials (487 ms) than on congruent trials $(472 \mathrm{~ms})$, resulting in a significant main effect of congruency. Congruency effects 

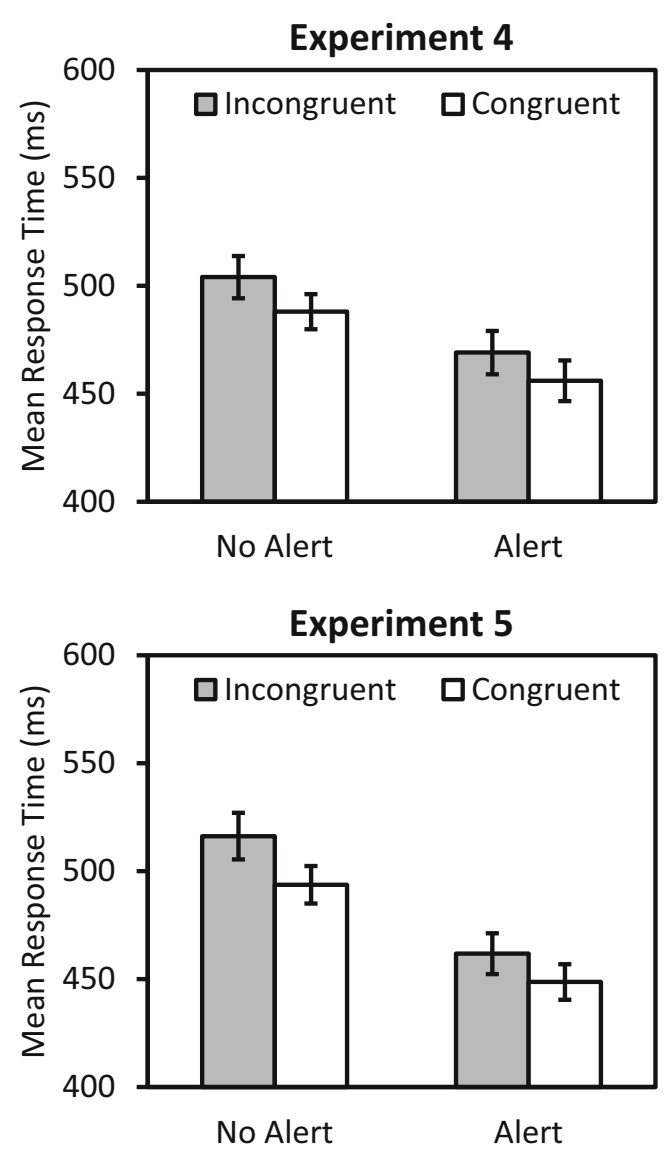

Fig. 3 Mean response times as a function of alerting and congruency in Experiments 4 and 5. Error bars represent standard errors of the means

were $13 \mathrm{~ms}$ and $16 \mathrm{~ms}$ for alert and no-alert trials, respectively, resulting in a nonsignificant interaction, $B F_{\text {null }}=10.3$.

Experiment 5 Subjects were faster on alert trials $(455 \mathrm{~ms})$ than on no-alert trials $(505 \mathrm{~ms})$, resulting in a significant main effect of alerting. They were slower on incongruent trials (489 ms) than on congruent trials $(471 \mathrm{~ms})$, resulting in a significant main effect of congruency. Congruency effects were $13 \mathrm{~ms}$ and $23 \mathrm{~ms}$ for alert and no-alert trials, respectively, resulting in a significant interaction $(p=.037$; see Table 2$)$, $B F_{\text {null }}=1.4$.

\section{Discussion}

Experiments 4 and 5 produced similar patterns of results (see Fig. 3). Subjects were faster on alert trials than on no-alert trials, replicating the alerting effects found in Experiments $1-3$. They were slower on incongruent trials than on congruent trials, indicating that congruency effects can be obtained in the color-word Stroop task even when the relevant and irrelevant stimulus features are spatially separated, consistent with previous findings (e.g., Gatti \& Egeth, 1978). In Experiment 4 , congruency effects were similar on alert and no-alert trials, resulting in a nonsignificant alerting-congruency interaction and a Bayes factor that strongly favored no difference in congruency effects. There was a significant alerting-congruency interaction in Experiment 5, but the form of the interaction was in the opposite direction - a smaller congruency effect on alert trials than on no-alert trials - of the alerting-congruency interaction typically found in the arrow flanker task, and it was in line with what would be expected for a scaling effect. However, the interaction was associated with a Bayes factor that weakly favored no difference in congruency effects. Experiments 4 and 5 provide evidence that spatial separation of relevant and irrelevant stimulus features is insufficient for producing a typical alerting-congruency interaction in the Stroop task.

These results seem problematic for theoretical accounts based on diffuse attention (McConnell \& Shore, 2011; Nieuwenhuis \& de Kleijn, 2013; Weinbach \& Henik, 2012). If increased alertness triggers a wider focus of attention, or if attention is initially more diffuse on alert trials, then the distractor words should have been attended and processed to a greater extent on alert trials than on no-alert trials in Experiments 4 and 5. The absence of a typical alerting-congruency interaction in both experiments is inconsistent with that expectation. The results are less problematic for the idea that increased alertness promotes more spatial grouping of targets and distractors (Schneider, 2018b). Based on the Gestalt law of perceptual organization by similarity (for an overview, see Palmer, 1999), the dissimilarity between the target stimuli ( $X X X X$ or a rectangle) and distractors (GREEN and $R E D$ ) for the Stroop task in Experiments 4 and 5 likely would have led to a low probability of spatial grouping regardless of alertness, in contrast with the high similarity between the targets and distractors in the arrow flanker task.

The results of Experiments 4 and 5, in conjunction with those of Experiments 1-3, help to clarify the nature of a possible spatial attention constraint on the relationship between alertness and cognitive control. Spatial separation versus integration of stimulus features did not determine the presence of a typical alerting-congruency interaction, suggesting that the constraint does not reflect the division of spatial attention between different stimuli in the visual field.

This suggestion and my results are inconsistent with a conclusion drawn by Weinbach and Henik (2012). After their aforementioned Experiment 2, which involved the colorword Stroop task, they investigated the issue of spatial integration versus separation of stimulus features in their Experiments 3 and 4. In those experiments, subjects performed a Stroop-like task that involved classifying the physical color (green or red) of a target stimulus accompanied by left-pointing or right-pointing distractor arrows that matched (congruent) or mismatched (incongruent) the left/right keypress response to the target. In their Experiment 3, the relevant and irrelevant stimulus features were spatially integrated 
because the target stimulus was a colored arrow. In their Experiment 4, the relevant and irrelevant stimulus features were spatially separated because the target stimulus was a colored rectangle flanked horizontally by distractor arrows. Their Experiment 3 yielded results that were similar to those of their Experiment 2, with significant main effects of alerting and congruency, but a nonsignificant alerting-congruency interaction. Critically, their Experiment 4 yielded a significant alerting-congruency interaction, similar in form to that typically found in the arrow flanker task. Considering that the experiments differed with respect to whether the relevant and irrelevant stimulus features were spatially integrated or separated, the contrasting results for the interaction led Weinbach and Henik to write:

Taken together, the results of Experiment $[\mathrm{s}] 3$ and 4 are conclusive. Alertness does not interfere in a response selection task when the relevant and irrelevant information are spatially integrated. The mere separation of the relevant and irrelevant features results in a greater congruency effect following alerting cues.... [This] finding suggests that the critical mechanism responsible for the appearance of the interaction is modulation of spatial attention following alerting cues. (Weinbach \& Henik, 2012, p. 1537)

As noted earlier, my results are inconsistent with this conclusion, motivating attempts to resolve the inconsistency between studies. My first attempt was based on the observation that the irrelevant stimulus features in my Experiments 1-5 were color words, whereas they were arrows in Experiments 3 and 4 of Weinbach and Henik (2012). Arrows have wellestablished spatial connotations that can influence spatial attention, even when the arrows are distinct from the target of the task (e.g., Hommel, Pratt, Colzato, \& Godijn, 2001; Tipples, 2002). In contrast, color words are not strongly associated with spatial directions; therefore, they would be unlikely to influence spatial attention, regardless of whether they are spatially integrated with or separated from the target. This line of reasoning led me to wonder whether the nature of the spatial attention constraint on the relationship between alertness and cognitive control is twofold: (a) The relevant and irrelevant stimulus features need to be spatially separated, and (b) the irrelevant stimulus features need to convey spatial information.

This dual constraint would fully accommodate the results from my experiments (to this point) and those of Weinbach and Henik (2012). The recurring absence of typical alertingcongruency interactions in my Experiments 1-5 would be due to the irrelevant stimulus features being color words that did not convey spatial information. Weinbach and Henik's findings of significant alerting-congruency interactions in their Experiments 1 and 4 would be due to the stimulus features being spatially separated and the irrelevant stimulus features being arrows that conveyed spatial information. The null alerting-congruency interactions in Weinbach and Henik's Experiments 2 and 3 would be due to the stimulus features being spatially integrated. Thus, the dual-constraint idea seems to have merit, and I tested it in the remaining experiments of the present study.

In Experiments 6 and 7, subjects performed a Stroop-like task that involved making a left/right key-press response to classify the physical color (green or red) of a target stimulus that was flanked above and below by distractors that were white-colored spatial words (LEFT or RIGHT). Experiments 6 and 7 were otherwise identical to Experiments 4 and 5, respectively, with the target stimulus being a colored letter string $(X X X X)$ in Experiment 6 and a colored rectangle in Experiment 7. An example stimulus for each experiment is shown in Fig. 1. Analogous to previous experiments, congruency was defined by the match (congruent) or mismatch (incongruent) between the spatial response to the target color and the meaning of the distractor word. I used spatial words instead of arrows as the distractors in these experiments to facilitate comparison with my earlier experiments (which also involved words as the distractors) and to avoid the confound of simultaneously changing the stimulus format (word vs. arrow) and the type of information conveyed by the distractors (color vs. spatial). Most importantly, the dual constraint was present in Experiments 6 and 7: (a) The relevant and irrelevant stimulus features were spatially separated, and (b) the irrelevant stimulus features conveyed spatial information. If the relationship between alertness and cognitive control depends on this dual constraint, then one would expect to find the typical alerting-congruency interaction.

\section{Experiments 6 and 7}

\section{Method}

Subjects Each experiment involved 86 students (Experiment 6: 52 female, 78 right-handed, mean age $=19.0$ years; Experiment 7: 48 female, 79 right-handed, mean age $=19.3$ years).

Task and stimuli Subjects performed a Stroop-like task that involved classifying the physical color (green or red) of a centrally presented target stimulus that was flanked by identical distractor spatial words (LEFT or RIGHT) above and below it. The physical color of the distractor words was always white. In Experiment 6, the target stimulus was a colored letter string $(X X X X)$, whereas in Experiment 7 , it was a colored rectangle. All four possible combinations of target color and distractor word were used, resulting in stimulus displays that were either congruent (target-color spatial response and 
distractor word meaning matched; e.g., red $X X X X$ or red rectangle - requiring a right-key response-flanked by RIGHT) or incongruent (target-color spatial response and distractor word meaning mismatched; e.g., green $X X X X$ or green rectangle-requiring a left-key response-flanked by RIGHT; see Fig. 1), with the examples assuming that green and red colors are mapped to left-key and right-key responses, respectively.

Procedure The procedure was identical to that of Experiments 3-5.

\section{Results}

Data trimming and statistical analyses followed those of Experiments 1-5. Mean RTs and error rates as a function of alerting and congruency appear in Table 1. The ANOVA results are summarized in Table 2. The RT data are presented visually in Fig. 4.

Experiment 6 Subjects were faster on alert trials (467 ms) than on no-alert trials $(507 \mathrm{~ms})$, resulting in a significant main effect of alerting. They were slower on incongruent trials (495 $\mathrm{ms}$ ) than on congruent trials $(478 \mathrm{~ms})$, resulting in a
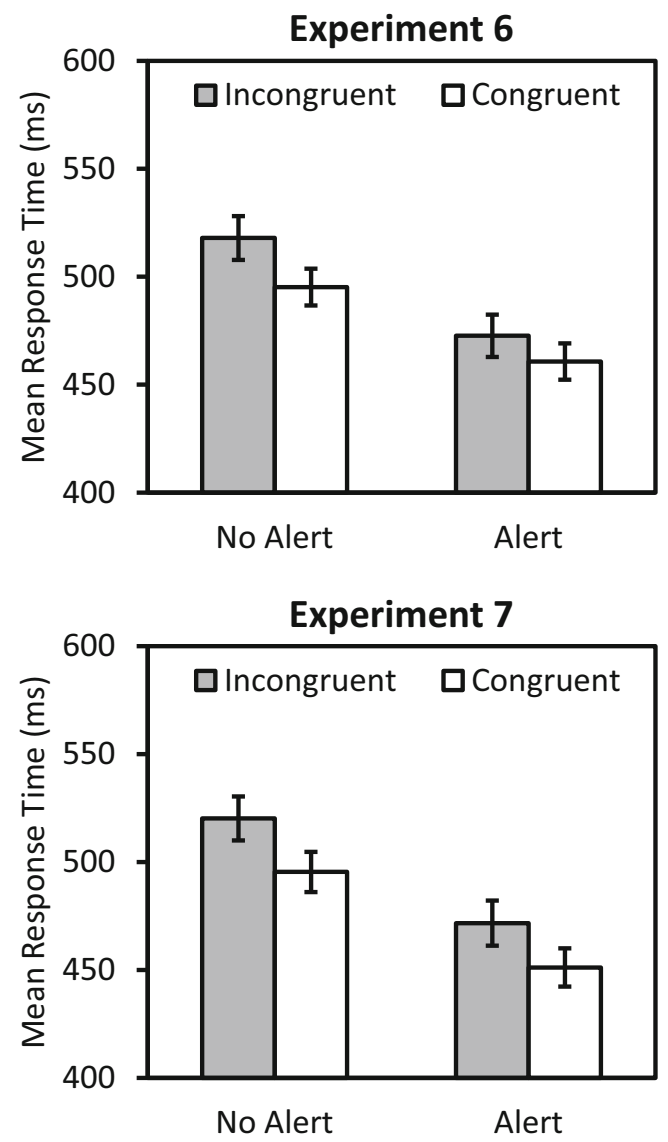

Fig. 4 Mean response times as a function of alerting and congruency in Experiments 6 and 7. Error bars represent standard errors of the means. significant main effect of congruency. Congruency effects were $12 \mathrm{~ms}$ and $23 \mathrm{~ms}$ for alert and no-alert trials, respectively, resulting in a significant interaction ( $p=.010$; see Table 2$)$, $B F_{\text {alt }}=2.3$.

Experiment 7 Subjects were faster on alert trials (461 ms) than on no-alert trials $(508 \mathrm{~ms})$, resulting in a significant main effect of alerting. They were slower on incongruent trials (496 ms) than on congruent trials $(473 \mathrm{~ms})$, resulting in a significant main effect of congruency. Congruency effects were $21 \mathrm{~ms}$ and $25 \mathrm{~ms}$ for alert and no-alert trials, respectively, resulting in a nonsignificant interaction, $B F_{\text {null }}=7.5$.

\section{Discussion}

Experiments 6 and 7 produced similar patterns of results (see Fig. 4). Subjects were faster on alert trials than on no-alert trials, replicating the alerting effects found in Experiments 1-5. They were slower on incongruent trials than on congruent trials, indicating that congruency effects can be obtained in a Stroop-like task in which the relevant stimulus feature is physical color and the irrelevant stimulus features are spatial (rather than color) words. There was a significant alertingcongruency interaction in Experiment 6, but as in Experiment 5, the form of the interaction was in the opposite direction - a smaller congruency effect on alert trials than on no-alert trials - of the alerting-congruency interaction typically found in the arrow flanker task, and it was in line with what would be expected for a scaling effect. The interaction was associated with a Bayes factor that weakly favored a difference in congruency effects. In Experiment 7, congruency effects were similar on alert and no-alert trials, resulting in a nonsignificant alerting-congruency interaction and a Bayes factor that moderately favored no difference in congruency effects. Experiments 6 and 7 provide evidence that spatial separation of relevant and irrelevant stimulus features, coupled with irrelevant stimulus features that convey spatial information, is insufficient for producing a typical alertingcongruency interaction in a Stroop-like task.

These results do not support the idea of a dual constraint on the relationship between alertness and cognitive control. The lack of support does not necessarily seem attributable to the use of spatial words as the irrelevant stimulus features because the congruency manipulation was very effective, yielding highly significant congruency effects (see Table 2). However, spatial words do not convey spatial information in the same way that arrows do, which might affect whether a typical alerting-congruency interaction is elicited. Recall that Weinbach and Henik (2012) obtained a significant alertingcongruency interaction in their Experiment 4, in which subjects performed a Stroop-like task on a colored target rectangle flanked by distractor arrows. This raises the possibility that a typical alerting-congruency interaction might be detectable if 
the irrelevant stimulus features are arrows instead of spatial words.

In Experiment 8, subjects performed a Stroop-like task that involved making a left/right key-press response to classify the physical color (green or red) of a target rectangle that was flanked horizontally by identical white-colored distractor arrows (two on each side on the target). An example stimulus is shown in Fig. 1. Experiment 8 was otherwise identical to Experiments 4-7. Analogous to Experiments 6 and 7, congruency was defined by the match (congruent) or mismatch (incongruent) between the spatial response to the target color and the direction of the distractor arrows. I chose to conduct a single experiment involving a colored rectangle as the target stimulus (without a companion experiment involving colored $X X X X$ as the target stimulus) for two reasons. First, the results of Experiments 4-7 show that the two types of target stimuli produce similar data patterns. Second, Experiment 8 was designed to be a conceptual replication of Experiment 4 from Weinbach and Henik (2012; see footnote 4), albeit with a larger sample size and the same design modifications noted earlier (no neutral stimulus, variable fixation interval, and visual alerting cues). Weinbach and Henik's finding of a significant alerting-congruency interaction in the typical direction in their Experiment 4 led me to expect a similar finding in my Experiment 8.

\section{Experiment 8}

\section{Method}

Subjects The experiment involved 86 students ( 39 female, 76 right-handed, mean age $=19.7$ years). The preregistered sample size (see footnote 4 ) was selected to remain consistent with the sample sizes in Experiments 1-7. However, I also conducted power analyses using the mean RTs, standard deviations, and correlations between conditions from Experiment 4 of Weinbach and Henik (2012). ${ }^{8}$ Simulations indicated that 86 subjects would provide over $99.8 \%$ power to detect their observed alerting-congruency interaction, which reflected a 21$\mathrm{ms}$ difference in congruency effects on RTs between alert and no-alert trials $(d=0.55)$. Figure 5 shows the power to detect a range of interaction effect sizes (determined by systematically changing the magnitude of the congruency effect on alert trials) for my sample size $(N=86)$ and for Weinbach and Henik's sample size $(N=24)$. The results indicate that 86 subjects provide high power to detect weaker interactions than the original effect size (e.g., $80 \%$ power for $d=0.31$ ).

Task and stimuli Subjects performed a Stroop-like task that involved classifying the physical color (green or red) of a

\footnotetext{
${ }^{8}$ I thank Noam Weinbach for providing the data used in these power analyses.
}

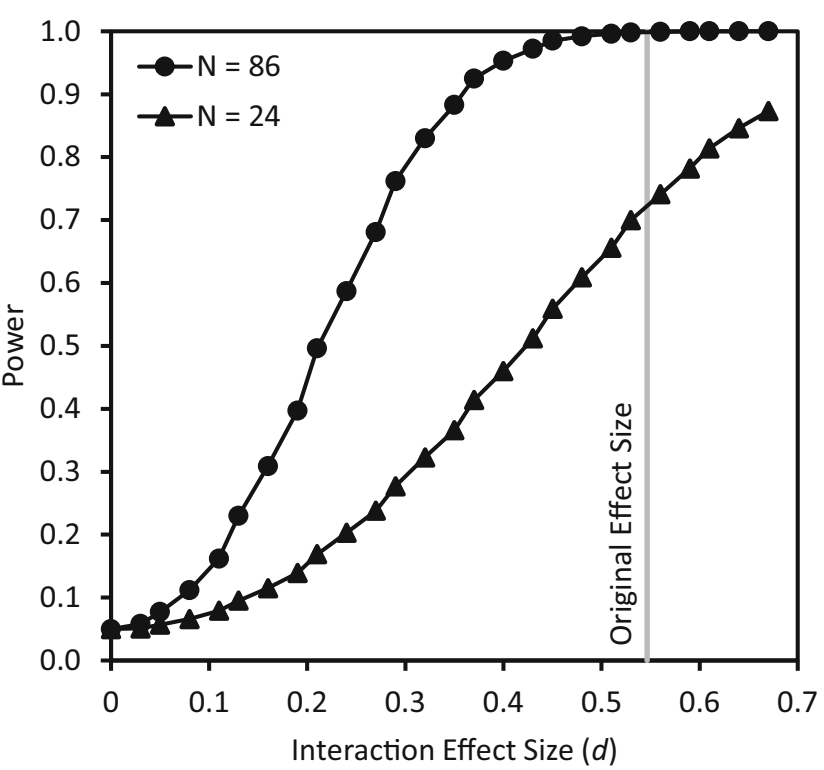

Fig. 5 Power analysis results for Experiment 8. Each curve indicates the power to detect various effect sizes for the interaction between alerting and congruency, assuming a specific sample size $(N)$. The line labeled "Original Effect Size" indicates the interaction effect size obtained in Experiment 4 of Weinbach and Henik (2012)

centrally presented target stimulus that was flanked by identical distractor arrows (pointing left or right), two on the left and two on the right of it. The target stimulus was a colored rectangle that was smaller $(0.8 \mathrm{~cm}$ wide) than in Experiments 5 and 7 to reduce the horizontal extent of the stimulus display and to more closely approximate the size of the target stimulus in Weinbach and Henik (2012). The distractor arrows were always white and measured $0.4 \mathrm{~cm}$ high $\times 0.8 \mathrm{~cm}$ wide. The center-to-center distance between adjacent stimuli was $1.0 \mathrm{~cm}$. All four possible combinations of target color and distractor arrow were used, resulting in stimulus displays that were either congruent (target-color spatial response and distractor arrow direction matched; e.g., red rectangle - requiring a rightkey response - flanked by right-pointing arrows) or incongruent (target-color spatial response and distractor arrow direction mismatched; e.g., green rectangle - requiring a left-key response-flanked by right-pointing arrows; see Fig. 1), with the examples assuming that green and red colors are mapped to left-key and right-key responses, respectively.

Procedure The procedure was identical to that of Experiments 3-7.

\section{Results}

Data trimming and statistical analyses followed those of Experiments 1-7. Mean RTs and error rates as a function of alerting and congruency appear in Table 1. The ANOVA results are summarized in Table 2. The RT data are presented visually in Fig. 6. 


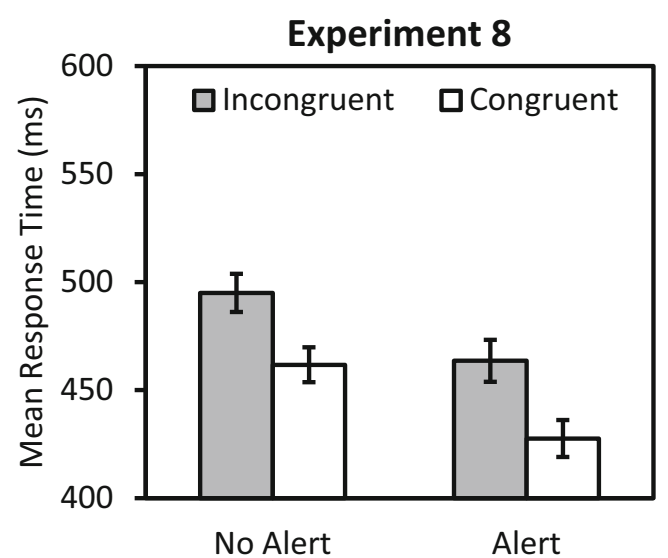

Fig. 6 Mean response times as a function of alerting and congruency in Experiment 8. Error bars represent standard errors of the means.

Subjects were faster on alert trials $(446 \mathrm{~ms})$ than on no-alert trials $(478 \mathrm{~ms})$, resulting in a significant main effect of alerting. They were slower on incongruent trials $(479 \mathrm{~ms})$ than on congruent trials (445 ms), resulting in a significant main effect of congruency. Congruency effects were $36 \mathrm{~ms}$ and $33 \mathrm{~ms}$ for alert and no-alert trials, respectively, resulting in a nonsignificant interaction, $B F_{\text {null }}=9.3$.

\section{Discussion}

Experiment 8 produced a notable pattern of results (see Fig. 6). Subjects were faster on alert trials than on no-alert trials, replicating the alerting effects found in Experiments 1-7. They were slower on incongruent trials than on congruent trials, indicating that congruency effects can be obtained in a Stroop-like task in which the relevant stimulus feature is physical color and the irrelevant stimulus features are arrows. Congruency effects were similar on alert and no-alert trials, resulting in a nonsignificant alerting-congruency interaction and a Bayes factor that moderately favored no difference in congruency effects. Collectively, the results of Experiment 8 only partially replicate those of Experiment 4 from Weinbach and Henik (2012). Both experiments yielded significant main effects of alerting and congruency, but my experiment did not replicate the alerting-congruency interaction that was significant in Weinbach and Henik's experiment.

It is unclear why the experiments produced different results for the interaction. As noted earlier, I regard my Experiment 8 as a conceptual replication of Experiment 4 from Weinbach and Henik (2012) because I made modifications to their experiment design (no neutral stimulus, variable fixation inter$\mathrm{val}$, and visual alerting cues). It is not obvious that the design modifications were responsible for the discrepancy between results, because I have consistently obtained significant alerting-congruency interactions with those design elements in previous experiments involving arrow flanker tasks (Schneider, 2018a, 2018b). Moreover, a variable fixation interval and visual alerting cues are commonly used in the ANT, where significant alerting-congruency interactions have been obtained (e.g., Fan et al., 2002; McConnell \& Shore, 2011; Redick \& Engle, 2006). My alerting and congruency manipulations did not appear to be less effective than Weinbach and Henik's manipulations, considering the similar-sized raw effects of alerting $(33 \mathrm{~ms}$ in my Experiment 8 vs. $44 \mathrm{~ms}$ in their Experiment 4) and congruency $(35 \mathrm{~ms}$ vs. $36 \mathrm{~ms})$ in the RT data. It seems unlikely that the null interaction in my experiment reflected a lack of statistical power, given that my sample size was more than 3 times as large as Weinbach and Henik's sample size, and it provided high power to detect even relatively small interaction effects (see Fig. 5). In addition, the Bayes factor for the interaction in my experiment moderately favored no difference in congruency effects between alert and no-alert trials $\left(B F_{\text {null }}=9.3\right.$; for comparison, $B F_{\text {alt }}=3.2$ for Weinbach and Henik's data). Thus, my tentative conclusion is that the alerting-congruency interaction is not robust in Stroop-like tasks that involve responding to colored target rectangles flanked by distractor arrows.

\section{General discussion}

The purpose of the present study was to investigate the generality of a puzzling interaction between alertness and cognitive control. At an empirical level, the interaction takes the form of a larger congruency effect on alert trials than on noalert trials in variants of the ANT used to study multiple components of attention (e.g., Callejas et al., 2005; Fan et al., 2002; MacLeod et al., 2010; McConnell \& Shore, 2011; Redick \& Engle, 2006; Schneider, 2018a, 2018b; Weinbach \& Henik, 2012). At a theoretical level, most explanations of the interaction can be divided into two groups, based on whether they involve mechanisms related to spatial attention (McConnell \& Shore, 2011; Nieuwenhuis \& de Kleijn, 2013; Schneider, 2018b; Weinbach \& Henik, 2012) or not (Callejas et al., 2005; Fischer et al., 2010, 2012). Considering the role of spatial attention in the arrow flanker task at the heart of the ANT, as well as in some of the theoretical accounts of the interaction, I was interested in whether there is a spatial attention constraint linking alertness to cognitive control.

To address this issue, I conducted a series of eight experiments (total $N=688$ ) in which I investigated alerting effects on the performance of Stroop or Stroop-like tasks with different kinds of stimuli (see Fig. 1). In Experiments 1-3, subjects performed a color-word Stroop task with spatially integrated stimuli that were congruent or incongruent (e.g., the word $R E D$ presented in green), with and without a visual alerting cue presented in advance (alert and no-alert trials, respectively). Subjects were consistently faster on alert trials than on noalert trials, indicating that the alerting cues were effective for 
increasing alertness. Subjects were consistently slower on incongruent trials than on congruent trials, indicating that the irrelevant stimulus feature (word meaning) was processed and influenced task performance. Critically, congruency effects were similar on alert and no-alert trials, resulting in nonsignificant alerting-congruency interactions and Bayes factors favoring no differences in congruency effects. These findings replicate the null interactions obtained in two previous experiments involving color-word Stroop tasks with spatially integrated stimuli (Soutschek et al., 2013, Weinbach \& Henik, 2012) and suggest that there is indeed a spatial attention constraint on the relationship between alertness and cognitive control.

The remaining experiments involved attempts to identify aspects of the spatial attention constraint by systematically modifying the Stroop task to include spatial elements related to those in the arrow flanker task. In Experiments 4 and 5, subjects performed a color-word Stroop task with spatially separated stimuli (e.g., a green rectangle flanked above and below by RED). Alerting and congruency effects were obtained, but a typical alerting-congruency interaction was not, suggesting that spatial separation of relevant and irrelevant stimulus features is insufficient for producing the interaction. In Experiments 6 and 7, subjects performed a Stroop-like task with spatially separated stimuli consisting of colored targets flanked by spatial words (e.g., a green rectangle flanked above and below by RIGHT). Once again, alerting and congruency effects were obtained, but a typical alerting-congruency interaction was not, suggesting that spatial separation of relevant and irrelevant stimulus features, coupled with irrelevant stimulus features that convey spatial information, is insufficient for producing the interaction. In Experiment 8, subjects performed a Stroop-like task with spatially separated stimuli consisting of colored target rectangles flanked horizontally by distractor arrows (e.g., a green rectangle flanked by rightpointing arrows). There were alerting and congruency effects, but no alerting-congruency interaction, which only partially replicates previous findings (Weinbach \& Henik, 2012).

Collectively, the results of Experiments 1-8 suggest that the alerting-congruency interaction typically found with the arrow flanker task used in the ANT does not generalize to Stroop or Stroop-like tasks that also involve cognitive control. Instead, there appears to be a spatial attention constraint that links alertness to cognitive control in a way that produces the interaction in the arrow flanker task. Before elaborating on the nature of the constraint, I discuss statistical and methodological considerations related to the issue that my conclusions are based on a series of (mostly) null interactions.

\section{Statistical and methodological considerations}

From a statistical perspective, the logic of null hypothesis significance testing (NHST) dictates that a nonsignificant result does not allow a researcher to conclude that an effect is absent. Instead, one can merely state that there is insufficient evidence of an effect, resulting in a failure to reject the null hypothesis of no effect. My response to this interpretive issue is threefold. First, power analyses indicated that my sample size per experiment $(N=86)$ provided high power to detect moderately sized alerting-congruency interactions (see Fig. 5). If the typical interaction exists with Stroop-like tasks and I simply did not detect it (making a Type II error), then it is likely a small effect. Second, Bayes-factor analyses circumvent the limitations of NHST, allowing researchers to quantify the evidence for or against the null hypothesis (Rouder et al., 2009). As indicated in the preceding Results sections, most of my Bayes factors strongly or moderately favored null interactions. Third, I conducted a random-effects meta-analysis of the interaction effects in the present experiments $(k=8, Q=$ $\left.8.2, I^{2}=15.0 \%\right)$, following the procedure described by Cumming (2012). The results are shown in the top panel of Fig. 7, where the interaction effect size (Cohen's $d$ ) is plotted separately for each experiment and for the set of experiments. Most of the $95 \%$ confidence intervals (CIs) include $d=0$, except for Experiments 5 and 6, which yielded significant
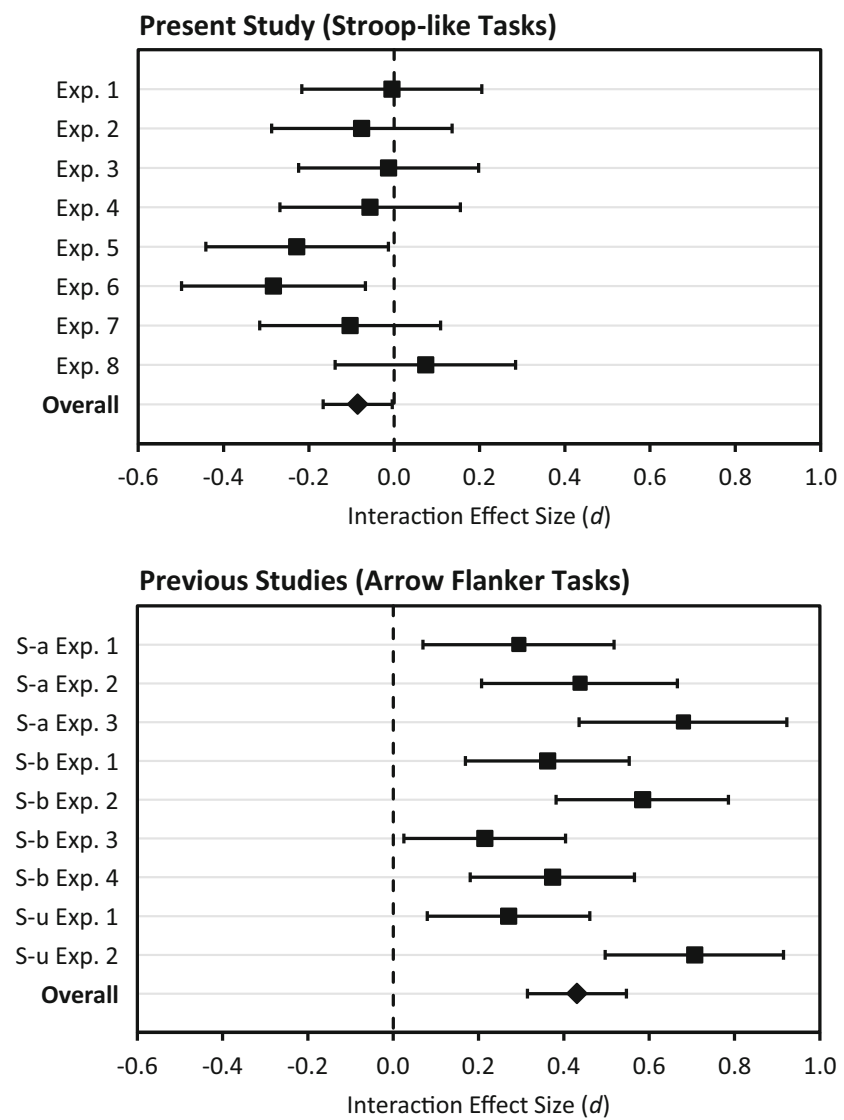

Fig. 7 Meta-analysis results for the effect size of the interaction between alerting and congruency in the present study (top panel) and in previous studies (bottom panel). Error bars represent $95 \%$ confidence intervals. Exp. $=$ Experiment S-a = Schneider (2018a); S-b = Schneider $(2018 b)$; S-u $=$ Schneider (2018c) 
interactions in atypical directions. The upper bounds of the CIs rarely extend beyond $d=0.2$, which is considered a small effect size (Cohen, 1988). Interestingly, the overall effect size for the set of experiments was significant $(d=-0.085,95 \% \mathrm{CI}$ $[-0.167,-0.004])$, but the direction of the effect was in line with a scaling effect, which is opposite of the typical alertingcongruency interaction.

From a methodological perspective, the recurrent finding of null alerting-congruency interactions with Stroop and Stroop-like tasks might make one wonder whether I can detect a typical alerting-congruency interaction at all, even with tasks that have been shown in previous studies to routinely produce the interaction (e.g., the arrow flanker task of the ANT). Perhaps there is something peculiar about my subject population or laboratory apparatus that impedes detection of alerting-congruency interactions. However, this concern is mitigated by my prior work on alertness and cognitive control. At the time of this writing, I have conducted nine experiments (total $N=902$ ) involving alerting manipulations in arrow flanker tasks. Seven of the experiments are published (Schneider, 2018a, 2018b) and two are currently unpublished (Schneider, 2018c). There are methodological differences across the experiments, but all involved the task of making a left/right key-press response to classify the left/ right direction of a central target arrow flanked by distractor arrows. As in the present study, the experiments involved visual alerting cues, variable fixation intervals, and no neutral stimuli. In four of the experiments, the alerting cue was a small square presented centrally at fixation, similar to the cue used in the present study. I conducted a random-effects meta-analysis of the interaction effects in my past experiments $(k=9, Q$ $=22.9, I^{2}=65.1 \%$ ), following the same procedure used earlier. The results are shown in the bottom panel of Fig. 7. None of the $95 \%$ CIs include $d=0$, because all nine experiments yielded significant alerting-congruency interactions in the typical direction. ${ }^{9}$ The overall effect size for the set of experiments was medium and significant $(d=0.430,95 \% \mathrm{CI}$ $[0.314,0.547])$. As noted earlier, the sample size used in the present experiments $(N=86)$ provides estimated power of $80 \%$ to detect a relatively small interaction effect size of $d=$

\footnotetext{
${ }^{9}$ Some readers might wonder about the probability of obtaining significant effects in all nine experiments, in light of analyses of the literature (e.g., Francis, 2014; Francis, Tanzman, \& Matthews, 2014) that have revealed a prevalence of excess success in psychology studies (i.e., more significant effects in a set of experiments than would be expected, based on estimates of effect size and power). This issue can be investigated with the test for excess significance (TES; Francis, 2012; Ioannidis \& Trikalinos, 2007), which yields the probability $\left(P_{\mathrm{TES}}\right)$ of obtaining significant effects in all the experiments in a set. A low probability $\left(P_{\mathrm{TES}}<.1\right.$ is a common criterion) indicates excess success. Applying the TES to my set of nine experiments yielded $P_{\mathrm{TES}}=.87$ ( or $P_{\mathrm{TES}}=.85$, if a fixed-effect meta-analytic effect size is used in the analysis). This result indicates that there is a high probability of obtaining significant effects in all nine experiments, mainly because every experiment had high power ( $>96 \%$, estimated using G*Power 3.1; Faul, Erdfelder, Lang, \& Buchner, 2007) to detect a medium-sized alerting-congruency interaction.
}

0.31, which corresponds to the lower bound of the $95 \% \mathrm{CI}$ around the overall effect size for arrow flanker tasks. The correspondence is coincidental, but it supports my earlier statistical points. In summary, from statistical and methodological perspectives, I think it is reasonable to conclude that my findings of null alerting-congruency interactions with Stroop and Stroop-like tasks can be interpreted as evidence of a spatial attention constraint linking alertness to cognitive control.

\section{The nature of the spatial attention constraint}

I mentioned in the Introduction section that the arrow flanker task at the heart of the ANT includes spatial separation of the target and distractors, stimuli (arrows) that have wellestablished spatial connotations, and spatially defined responses (left and right key presses). The present results for Stroop and Stroop-like tasks indicate that the spatial attention constraint is not whether the relevant and irrelevant stimulus features are spatially separated (Experiments 4-8) or integrated (Experiments 1-3), contrary to a conclusion drawn by Weinbach and Henik (2012). Even for spatially separated stimuli, the constraint is not whether the irrelevant stimulus features convey spatial information (Experiments 6-8) or not (Experiments 4 and 5). The use of spatially defined responses cannot be the (sole) constraint, because they were common to all the experiments in the present study.

The most salient differences that remain between Strooplike tasks and arrow flanker tasks are the type of target stimulus and the judgment performed on it. For Stroop-like tasks, the target stimulus feature (color) does not convey spatial information and subjects perform a perceptual judgment on it. For arrow flanker tasks, the target stimulus (arrow) conveys spatial information and subjects perform a spatial judgment on it. Previous research has shown that arrows are potent stimuli in visuospatial attention tasks, to the extent that they can automatically trigger orienting of attention, even when they are uninformative (e.g., Hommel et al., 2001; Tipples, 2002). It is possible that when target stimuli are nonspatial or lack wellestablished spatial associations, it is difficult to elicit a typical alerting-congruency interaction, even in the flanker task.

Some support for this point comes from studies involving variants of the ANT with nonarrow target stimuli. Rueda et al. (2004) developed a child-friendly version of the ANT in which the stimuli were line drawings of fish that faced left or right, with the target fish flanked by congruent or incongruent distractor fish. Congruency effects were obtained, but they were not reliably modulated by the cues that preceded the stimuli, despite evidence that the cues increased alertness (see also Ishigami \& Klein, 2015). Alerting-congruency interactions also appeared to be absent for children and adults who performed versions of the ANT with fish, schematic faces, or real faces as stimuli (Federico, Marotta, Adriani, 
Maccari, \& Casagrande, 2013; Federico, Marotta, Martella, \& Casagrande, 2017). ${ }^{10}$

However, there is evidence from other studies that the alerting-congruency interaction can be detected with nonarrow target stimuli. As described earlier, Weinbach and Henik (2012) found a significant alerting-congruency interaction with a colored target rectangle flanked by distractor arrows in their Experiment 4, but I did not replicate that finding in my Experiment 8. Seibold (2018) obtained significant alerting-congruency interactions with target letters flanked by distractor arrows or letters, but only when the set of potential targets was small. Fischer et al. (2012) found significant alertingcongruency interactions in word flanker tasks, but only when the distractor words belonged to the same stimulus set as the target words. Thus, the data seem inconclusive regarding whether the spatial attention constraint involves a target stimulus that conveys spatial information. $^{11}$

The importance of the type of target stimulus might depend on the task goal. Given that the instructed goal for both Stroop-like and flanker tasks is to judge the target while ignoring the distractors, part of the spatial attention constraint might be that alertness influences cognitive control primarily when the task goal is associated with spatial information processing. For tasks that involve cognitive control but have goals that are not intrinsically spatial (e.g., classifying colors), increased alertness might only facilitate stimulus encoding. For tasks that have spatial goals (e.g., classifying spatial directions), increased alertness might affect multiple stages of information processing (e.g., stimulus encoding and response selection), especially when the target stimuli have well-established spatial connotations.

A spatial attention constraint that involves a task goal associated with spatial information processing has implications for existing theoretical accounts of the alertingcongruency interaction. The idea that increased alertness leads to inhibition of cognitive control (Callejas et al.,

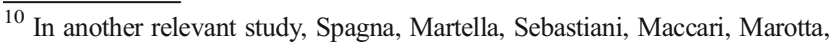
and Casagrande (2014) investigated a variant of the ANT in which two cues could each be present or absent during a trial: an auditory cue (a tone) and a visual cue (an asterisk appearing above or below fixation, validly or invalidly indicating the location of the forthcoming stimulus). They associated the auditory and visual cues with alerting and orienting functions, respectively, but it is reasonable to assume that the visual cues also modulated alertness. The task stimuli were arrows, fruits, or geometrical shapes. Spagna et al. found a significant alerting-congruency interaction linked to the auditory cues, but it did not differ across stimulus types. However, if the visual cues are also treated as alerting cues, then it is notable that their Fig. 3 shows a numerically larger congruency effect when the visual cues were present (averaging across valid and invalid cues) than when they were absent, but only for arrow stimuli.

${ }^{11}$ This point is complicated further by the recurrent finding of an alertingcongruency interaction in the Simon task (Böckler et al., 2011; Fischer et al., 2010; Klein \& Ivanoff, 2011; Soutschek et al., 2013), in which the target stimulus feature is usually nonspatial.
}

2005) is too vague to accommodate the present findings. To remain viable, it would need to be elaborated by specifying the mechanism underlying inhibition, including spatial attention constraints on the operation of that mechanism. A similar point can be made for the idea that increased alertness boosts activation of a direct route for response selection based on established stimulus-response associations (Fischer et al., 2010, 2012). It might be possible to retain that idea if it is made more specific by assuming that alertness only affects direct routes for spatial information processing.

There are also implications for theoretical accounts that already include spatial attention components. Explanations based on attention being more diffuse (at least initially) on alert trials than on no-alert trials (McConnell \& Shore, 2011; Nieuwenhuis \& de Kleijn, 2013; Weinbach \& Henik, 2012) need to be amended to account for my recurrent finding that spatial separation of relevant and irrelevant stimulus features is insufficient for producing the typical alerting-congruency interaction (Experiments 4-8). ${ }^{12}$ One possible amendment could involve changing the extent to which the distribution of spatial attention can be modulated, depending on the task goal (e.g., a task goal that is intrinsically spatial could allow greater modulation of spatial attention). This possibility is supported by previous work showing that the task goal can affect how spatial attention is distributed across an array of stimuli (e.g., LaBerge, 1983). A similar point can be made for the idea that increased alertness promotes more spatial grouping of targets and distractors (Schneider, 2018b) if the probability of spatial grouping is affected by the task goal.

The development and refinement of theoretical accounts of the relationship between alertness and cognitive control would benefit from additional studies involving tasks and stimuli that differ from the flanker task and arrow stimuli that are commonly used in the ANT. As demonstrated in the present study, alternative tasks and stimuli can reveal data patterns that point to constraints on the alerting-congruency interaction. Identifying these constraints is likely to be helpful in determining how alertness and cognitive control are connected within the human attention system.

Author note I thank Paige Asbridge, Timothy Durbin, Christine Grosso, Kelsey Lynch, Yijie Peng, Analicia Rios, and especially Sami Wagner for assistance with data collection. I also thank Sander Nieuwenhuis and two anonymous reviewers for their comments on this work.

\footnotetext{
12 These theoretical accounts are also challenged by other recent findings that do not support the idea that spatial attention is more diffuse on alert trials than on no-alert trials (Schneider, 2018b; Seibold, 2018).
} 


\section{References}

Böckler, A., Alpay, G., \& Stürmer, B. (2011). Accessory stimuli affect the emergence of conflict, not conflict control: A Simon-task ERP study. Experimental Psychology, 58, 102-109.

Callejas, A., Lupiáñez, J., Funes, M. J., \& Tudela, P. (2005). Modulations among the alerting, orienting, and executive control networks. Experimental Brain Research, 167, 27-37.

Callejas, A., Lupiáñez, J., \& Tudela, P. (2004). The three attentional networks: On their independence and interactions. Brain and Cognition, 54, 225-227.

Cohen, J. (1988). Statistical power analysis for the behavioral sciences (2nd ed.). Hillsdale, NJ: Erlbaum.

Cumming, G. (2012). Understanding the new statistics: Effect sizes, confidence intervals, and meta-analysis. New York, NY: Routledge.

Dunbar, K., \& MacLeod, C. M. (1984). A horse race of a different color: Stroop interference patterns with transformed words. Journal of Experimental Psychology: Human Perception and Performance, $10,622-639$.

Eriksen, B. A., \& Eriksen, C. W. (1974). Effects of noise letters upon the identification of a target letter in a nonsearch task. Perception \& Psychophysics, 16, 143-149.

Fan, J., McCandliss, B. D., Sommer, T., Raz, A., \& Posner, M. I. (2002). Testing the efficiency and independence of attentional networks. Journal of Cognitive Neuroscience, 14, 340-347.

Faul, F., Erdfelder, E., Lang, A.-G., \& Buchner, A. (2007). G*Power 3: A flexible statistical power analysis program for the social, behavioral, and biomedical sciences. Behavior Research Methods, 39, 175-191.

Federico, F., Marotta, A., Adriani, T., Maccari, L., \& Casagrande, M. (2013). Attention network test-The impact of social information on executive control, alerting and orienting. Acta Psychologica, 143, $65-70$.

Federico, F., Marotta, A., Martella, D., \& Casagrande, M. (2017). Development in attention functions and social processing: Evidence from the Attention Network Test. British Journal of Developmental Psychology, 35, 169-185.

Fischer, R., Plessow, F., \& Kiesel, A. (2010). Auditory warning signals affect mechanisms of response selection: Evidence from a Simon task. Experimental Psychology, 57, 89-97.

Fischer, R., Plessow, F., \& Kiesel, A. (2012). The effects of alerting signals in action control: Activation of S-R associations or inhibition of executive control processes? Psychological Research, 76, $317-328$

Francis, G. (2012). Publication bias and the failure of replication in experimental psychology. Psychonomic Bulletin \& Review, 19, 975991

Francis, G. (2014). The frequency of excess success for articles in Psychological Science. Psychonomic Bulletin \& Review, 21, 11801187.

Francis, G., Tanzman, J., \& Matthews, W. J. (2014). Excess success for psychology articles in the journal Science. PLOS ONE, 9(12), e114255.

Gatti, S. V., \& Egeth, H. E. (1978). Failure of spatial selectivity in vision. Bulletin of the Psychonomic Society, 11, 181-184.

Gratton, G., Coles, M. G. H., Sirevaag, E. J., Eriksen, C. W., \& Donchin, E. (1988). Pre- and poststimulus activation of response channels: A psychophysiological analysis. Journal of Experimental Psychology: Human Perception and Performance, 14, 331-344.

Heitz, R. P., \& Engle, R. W. (2007). Focusing the spotlight: Individual differences in visual attention control. Journal of Experimental Psychology: General, 136, 217-240.

Held, L., \& Ott, M. (2018). On p-values and Bayes factors. Annual Review of Statistics and Its Application, 5, 393-419.

Hommel, B., Pratt, J., Colzato, L., \& Godijn, R. (2001). Symbolic control of visual attention. Psychological Science, 12, 360-365.
Ioannidis, J. P. A., \& Trikalinos, T. A. (2007). An exploratory test for an excess of significant findings. Clinical Trials, 4, 245-253.

Ishigami, Y., \& Klein, R. M. (2015). Repeated measurement of the components of attention with young children using the Attention Network Test: Stability, isolability, robustness, and reliability. Journal of Cognition and Development, 16, 144-159.

Johnson, A., \& Proctor, R. W. (2004). Attention: Theory and practice. Thousand Oaks, CA: SAGE.

Klein, R. M., \& Ivanoff, J. (2011). The components of visual attention and the ubiquitous Simon effect. Acta Psychologica, 136, 225-234.

LaBerge, D. (1983). Spatial extent of attention to letters and words. Journal of Experimental Psychology: Human Perception and Performance, 9, 371-379.

Logan, G. D. (1996). The CODE theory of visual attention: An integration of space-based and object-based attention. Psychological Review, 103, 603-649.

Lu, C.-H., \& Proctor, R. W. (1995). The influence of irrelevant location information on performance: A review of the Simon and spatial Stroop effects. Psychonomic Bulletin \& Review, 2, 174-207.

Lukas, S., Philipp, A. M., \& Koch, I. (2010). The role of preparation and cue-modality in crossmodal task switching. Acta Psychologica, 134, 318-322.

MacLeod, C. M. (1991). Half a century of research on the Stroop effect: An integrative review. Psychological Bulletin, 109, 163-203.

MacLeod, J. W., Lawrence, M. A., McConnell, M. M., Eskes, G. A., Klein, R. M., \& Shore, D. I. (2010). Appraising the ANT: Psychometric and theoretical considerations of the Attention Network Test. Neuropsychology, 24, 637-651.

Mather, M., \& Sutherland, M. R. (2011). Arousal-biased competition in perception and memory. Perspectives on Psychological Science, 6, 114-133.

McClain, L. (1983). Effects of response type and set size on Stroop colorword performance. Perceptual and Motor Skills, 56, 735-743.

McConnell, M. M., \& Shore, D. I. (2011). Mixing measures: Testing an assumption of the attention network test. Attention, Perception, \& Psychophysics, 73, 1096-1107.

Navon, D. (1977). Forest before trees: The precedence of global features in visual perception. Cognitive Psychology, 9, 353-383.

Nieuwenhuis, S., \& de Kleijn, R. (2013). The impact of alertness on cognitive control. Journal of Experimental Psychology: Human Perception and Performance, 39, 1797-1801.

Open Science Collaboration. (2015). Estimating the reproducibility of psychological science. Science, 349(6251), aac4716.

Palmer, S. E. (1999). Vision science: Photons to phenomenology. Cambridge, MA: MIT Press.

Pashler, H. E. (1998). The psychology of attention. Cambridge, MA: MIT Press.

Petersen, S. E., \& Posner, M. I. (2012). The attention system of the human brain: 20 years after. Annual Review of Neuroscience, 35, 73-89.

Posner, M. I. (2008). Measuring alertness. Annals of the New York Academy of Sciences, 1129, 193-199.

Posner, M. I., \& Boies, S. J. (1971). Components of attention. Psychological Review, 78, 391-408.

Pratte, M. S., Rouder, J. N., Morey, R. D., \& Feng, C. (2010). Exploring the differences in distributional properties between Stroop and Simon effects using delta plots. Attention, Perception, \& Psychophysics, 72, 2013-2025.

Quinlan, P. T., \& Hill, N. I. (1999). Sequential effects in rudimentary auditory and visual tasks. Perception \& Psychophysics, 61, 375384.

Redick, T. S., \& Engle, R. W. (2006). Working memory capacity and attention network test performance. Applied Cognitive Psychology, $20,713-721$

Robertson, I. H., Mattingley, J. B., Rorden, C., \& Driver, J. (1998). Phasic alerting of neglect patients overcomes their spatial deficit in visual awareness. Nature, 395, 169-172. 
Rolke, B., \& Hofmann, P. (2007). Temporal uncertainty degrades perceptual processing. Psychonomic Bulletin \& Review, 14, 522-526.

Rouder, J. N., Speckman, P. L., Sun, D., Morey, R. D., \& Iverson, G. (2009). Bayesian $t$ tests for accepting and rejecting the null hypothesis. Psychonomic Bulletin \& Review, 16, 225-237.

Rueda, M. R., Fan, J., McCandliss, B. D., Halparin, J. D., Gruber, D. B., Pappert Lercari, L., \& Posner, M. I. (2004). Development of attentional networks in childhood. Neuropsychologia, 42, 1029-1040.

Schneider, D. W. (2018a). Alertness and cognitive control: Testing the early onset hypothesis. Journal of Experimental Psychology: Human Perception and Performance, 44, 756-766.

Schneider, D. W. (2018b). Alertness and cognitive control: Toward a spatial grouping hypothesis. Attention, Perception, \& Psychophysics, 80, 913-928.

Schneider, D. W. (2018c). [Spatial alignment effects in the flanker task]. Unpublished raw data.

Schönbrodt, F. D., \& Wagenmakers, E.-J. (2018). Bayes factor design analysis: Planning for compelling evidence. Psychonomic Bulletin \& Review, 25, 128-142.

Seibold, V. C. (2018). Do alerting signals increase the size of the attentional focus? Attention, Perception, \& Psychophysics, 80, 402-425.

Seifried, T., Ulrich, R., Bausenhart, K. M., Rolke, B., \& Osman, A. (2010). Temporal preparation decreases perceptual latency: Evidence from a clock paradigm. Quarterly Journal of Experimental Psychology, 63, 2432-2451.

Simon, J. R., \& Small, A. M. (1969). Processing auditory information: Interference from an irrelevant cue. Journal of Applied Psychology, $53,433-435$.

Smith, P. L., \& Ratcliff, R. (2015). Diffusion and random walk processes. In J. D. Wright (Ed.), International encyclopedia of the social and behavioral sciences (2nd ed., Vol. 6, pp. 395-401). Oxford, UK: Elsevier.
Soutschek, A., Müller, H. J., \& Schubert, T. (2013). Conflict-specific effects of accessory stimuli on cognitive control in the Stroop task and the Simon task. Experimental Psychology, 60, 140-147.

Spagna, A., Martella, D., Sebastiani, M., Maccari, L., Marotta, A., \& Casagrande, M. (2014). Efficiency and interactions of alerting, orienting and executive networks: The impact of imperative stimulus type. Acta Psychologica, 148, 209-215.

Spencer, K. M., \& Coles, M. G. H. (1999). The lateralized readiness potential: Relationship between human data and response activation in a connectionist model. Psychophysiology, 36, 364-370.

Stroop, J. R. (1935). Studies of interference in serial verbal reactions. Journal of Experimental Psychology, 18, 643-662.

Tipples, J. (2002). Eye gaze is not unique: Automatic orienting in response to uninformative arrows. Psychonomic Bulletin \& Review, 9, 314-318.

Wagenmakers, E.-J., \& Brown, S. (2007). On the linear relation between the mean and the standard deviation of a response time distribution. Psychological Review, 114, 830-841.

Warren, C. M., Murphy, P. R., \& Nieuwenhuis, S. (2016). Cognitive control, dynamic salience, and the imperative toward computational accounts of neuromodulatory function. Behavioral and Brain Sciences, 39, 45-46.

Weinbach, N., \& Henik, A. (2011). Phasic alertness can modulate executive control by enhancing global processing of visual stimuli. Cognition, 121, 454-458.

Weinbach, N., \& Henik, A. (2012). The relationship between alertness and executive control. Journal of Experimental Psychology: Human Perception and Performance, 38, 1530-1540.

Weinbach, N., \& Henik, A. (2014). Alerting enhances attentional bias for salient stimuli: Evidence from a global/local processing task. Cognition, 133, 414-419.

White, C. N., Ratcliff, R., \& Starns, J. J. (2011). Diffusion models of the flanker task: Discrete versus gradual attentional selection. Cognitive Psychology, 63, 210-238. 